\title{
ARITMÉTICAS EN UN MUNICIPIO DEL NORORIENTE COLOMBIANO
}

\section{ARITHMETIC IN A REGION OF NORTHEASTERN COLOMBIA}

\begin{abstract}
Armando Aroca ${ }^{1}$
${ }^{1}$ Lic. Matemáticas, Magister en Educación con énfasis en educación matemática. Estudiante del doctorado Interinstitucional de Educación Universidad Distrital Francisco José de Caldas. Profesor Asociado Universidad del Atlántico, Barranquilla, Colombia, km 7, antigua vía a Puerto Colombia, e-mail: armandoaroca@mail.uniatlantico.edu.co
\end{abstract}

Rev. U.D.C.A Act. \& Div. Cient. 18(2): 553-561, Julio-Diciembre, 2015

\section{RESUMEN}

Una aritmética existe por necesidad de una actividad humana y solo tiene sentido en el intercambio de los productos que genera o moviliza. El objetivo de esta investigación fue analizar las aritméticas en un sector del municipio de la Paz, departamento del Cesar, Colombia. Se identificaron, a la vez, los procesos y los algoritmos que emplean personas adultas, al hacer cálculos en algunos oficios y se determinó el grado de participación de los niños en estas actividades. He aquí justamente lo problemático, a que se hace referencia, cuando se dice que la matemática tiene sentido si se vincula con la realidad. La metodología empleada fue básicamente cualitativa de tipo etnográfica, cuyas estrategias de recolección de datos consistió en 17 entrevistas informales de periodos cortos, observaciones no participantes de actividades y el empleo del google earth, como herramienta de localización de la actividad, dentro de un mapa. Los resultados muestran que es posible determinar cuándo el niño o niña se vincula, de manera concreta, a las actividades de su entorno sociocultural próximo, en el sentido de comprender los procesos y los algoritmos de los cálculos empleados en ellas.

Palabras clave: Procesos, algoritmos, relación con la realidad, contexto sociocultural, contexto escolar, etnomatemáticas.

\section{SUMMARY}

An arithmetic exists for human activities and only makes sense for the exchange of the products produces or mobilized by it. The objective of this research was to analyze the arithmetic in a specific region of a small Colombian town. Both processes and algorithms being employed by adults to make calculations in some trades were identified and the degree of participation of children in these activities was determined, which could relate to what appeals in math classes as reality. Here there is aright the problem, referred to when mentioned that mathematics has sense if it is linked with reality. The methodology used was basically of qualitative ethnographic type, which strategies for data collection consisted of informal interviews, observations of activities and the use of google earth. The results show that it is possible to determine when a child is linked specifically to the activities of its surrounding sociocultural environment in the sense of understanding the processes and algorithms used in these calculations.

Key words: Arithmetic, relation to reality, socio-cultural context, school context, ethnomathematics.

\section{INTRODUCCIÓN}

Desde el comienzo de la historia moderna de las ciencias y de las matemáticas, en el siglo XIX y tomando como referencia algunos estudios sobre conocimiento humano y etnomatemática, realizados por D’Ambrosio (2000; 2011; 2012), se puede deducir que en todas las culturas la aritmética nace por la necesidad que tienen los seres humanos de observar, de comparar, de clasificar, de cuantificar, de medir, de ordenar y de inferir, tal como se puede deducir de Taylor (1816), de Boyer (1974), de Hodgkin (2005) y de Seaquist et al. (2005), que ha sido desde tiempos pre-históricos. La necesidad ha existido desde el momento en que la especie humana interactuó con su entorno, en principio geográfico y, luego, sociocultural. Una aritmética existe, debido a una actividad de la persona que la necesita y sólo tiene sentido en la transacción o intercambio de los productos que moviliza; como ejemplo, se pueden analizar los trabajos de Saxe (1988; 1991), quien analizó el conocimiento matemático de los vendedores ambulantes o de quienes ejercen sus oficios en las calles. A diferencia de la aritmética escolar, ésta no cobra sentido a partir de alguna actividad productiva o de 
intercambio concreto, sino por la necesidad de educar en masa. Sólo la aritmética escolar tiene como objetivo que otras personas la aprendan masivamente y la incorporen en su forma de vivir, lo que genera tensiones, dado que el estudiante pertenece a un contexto sociocultural específico, que le provee otras formas de hacer, de pensar y de comunicar aritméticas; experimenta lo que se podría denominar una falta de comprensión interlógica de las aritméticas. Por un lado, el contexto escolar privilegia el cálculo numérico por escrito, mientras que el sociocultural privilegia el mental, cuanto este último, es la base del primero. Por lo general, el contexto escolar tiene el foco en la enseñanza de las técnicas o algoritmos y no se establece relación con las otras aritméticas, surgidas a partir de las actividades socioculturales, en las que han participado los alumnos, en su vida cotidiana. Dichas tensiones, se podrían establecer de la siguiente manera: en primer lugar, los ámbitos socioculturales, no sólo determinan cómo se adquiere el conocimiento, sino también la forma en que se representa, organiza y conserva, de acuerdo con lo planteado por Chahine \& Naresh (2013); en segundo lugar, la lógica de una aritmética externa a la escolar no siempre puede entender a ésta en el acto; estas dos aritméticas, la escolar y de la calle no guardan algo en común, solo se encuentra entre ellas semejanzas de familia (Wittgenstein, 1997). Es una afirmación que entramos a cuestionar: como se verá más adelante el cálculo mental si les permite guardar algo en común y, en tercer lugar, el proceso escolar tiene como finalidad que la lógica de los algoritmos y las operaciones de la aritmética sea entendida y aplicada, pues si no tiene esta segunda finalidad, entonces, ¿para qué sirve la primera? A estas dos últimas tensiones, se llamarán tensiones de intercomprensión de lógicas.

\section{MATERIALES Y MÉTODOS}

Se tomó como muestra un sector del municipio de La Paz, departamento del Cesar, ubicado al norte de Colombia. Se escogió este sitio, porque se hicieron observaciones previas, que identificarán un lugar que aglutinará varios oficios o actividades, además que fuera una población conocida por el autor. Algunos de estos oficios o actividades son: ventas ambulantes de fritos y de leche, ventas de frutas o verduras en la plaza de mercado, albañilería, curtiembre artesanal del cuero, carpintería, zapatería, modistería, juegos infantiles, peluquería y algunos oficios de campesinos. A este sector, se le tomó una foto por medio de google earth (Figura 1). En algunos casos, fue necesario ir al mismo lugar donde se desarrollaba el oficio, como por ejemplo, la curtiembre artesanal del cuero, elaboración de mecedoras y carpintería; luego, se hizo, en algunas mañanas y tardes, varios recorridos por las calles de esta zona, para identificar ciertos oficios o actividades que se daban allí; por ello, en la figura 1, aparecen triángulos monocolor y bicolores; el impacto visual que se quería en esta figura no se logró empleando marcas de posi- ción de google earth, por eso se descartaron. Cada triángulo es la representación de un oficio que se consideró que tiene una aritmética asociada, con sus procesos y algoritmos. En la figura 2, se explica cada uno de estos triángulos. En algunos momentos, sobre todo en las tardes o fines de semana, se hacían observaciones no participantes desde las tiendas de barrios o casas de amigos, para establecer qué hacían los niños, de un rango de edad entre 8 a 12 años; estas edades, en algunos casos, fueron consultadas directamente con los menores y, en otros, fueron estimadas. También, se hacían estas observaciones para establecer qué oficios se daban en algunas partes del sector escogido, como calles, avenidas, manzanas y barrios; esto permitió hacer el primer mapeo aritmético. Dicho mapeo, se hizo dos veces y se notó que es temporal, es decir, cambiante. Algunos oficios tienden a ser más fijos, geográficamente hablando, otros se mueven de manera regular por una misma trayectoria, otros son espontáneos y dependen de algunas variables, por ejemplo, un juego de moda o la decisión de un grupo de niños por jugar tal juego, porque llovió, porque cuentan con terreno sin pavimentar o porque hay pavimento.

En otros casos, haciendo algunas entrevistas, se preguntó a los niños su edad, su grado de formación escolar y su ocupación en los tiempos libres. También, se hicieron algunas entrevistas, conversaciones, a padres sobre las ocupaciones de sus hijos en su tiempo no escolar, al igual que entrevistas o conversaciones informales con adultos, que ejercían los oficios que se describen en la figura 2. Por lo anterior, es que se estableció en el resumen, que la investigación es cualitativa, tomando como referencia Deslauriers (2005) y que es de tipo etnográfica (Morse et al. 2007; Goetz \& LeCompte, 1998). El análisis de datos estuvo en función de la construcción de categorías emergentes, que se van mostrando a medida que aparecen las figuras de este artículo (Strauss \& Corbin, 2002). En cuanto al margen de error de la figura 1, se ha precisado que las actividades u oficios del ser humano, cambian, mutan, algunas son fijas, en términos geográficos y, otras no; es por ello, que dicho margen no es sencillo precisar. Se trató de emplear el mayor rigor metodológico, ya descrito, para dar una aproximación de la realidad.

\section{RESULTADOS Y DISCUSIÓN}

Se encontró, como ocurre también en el contexto escolar, que los niños necesitan cierta edad o ciertas competencias cognoscitivas para poder comprender algunos algoritmos u operaciones empleados en diversos oficios de la calle, los cuales, nadie se los enseña, sino que las aprenden por experiencia, por tensiones inherentes a la comunicación social, por necesidad o por tradición de oficio familiar. La figura 1 muestra una aproximación de la ubicación de oficios que se consideraron emplean su propia aritmética; son apenas una aproximación de la diversidad de oficios que se presentan en 


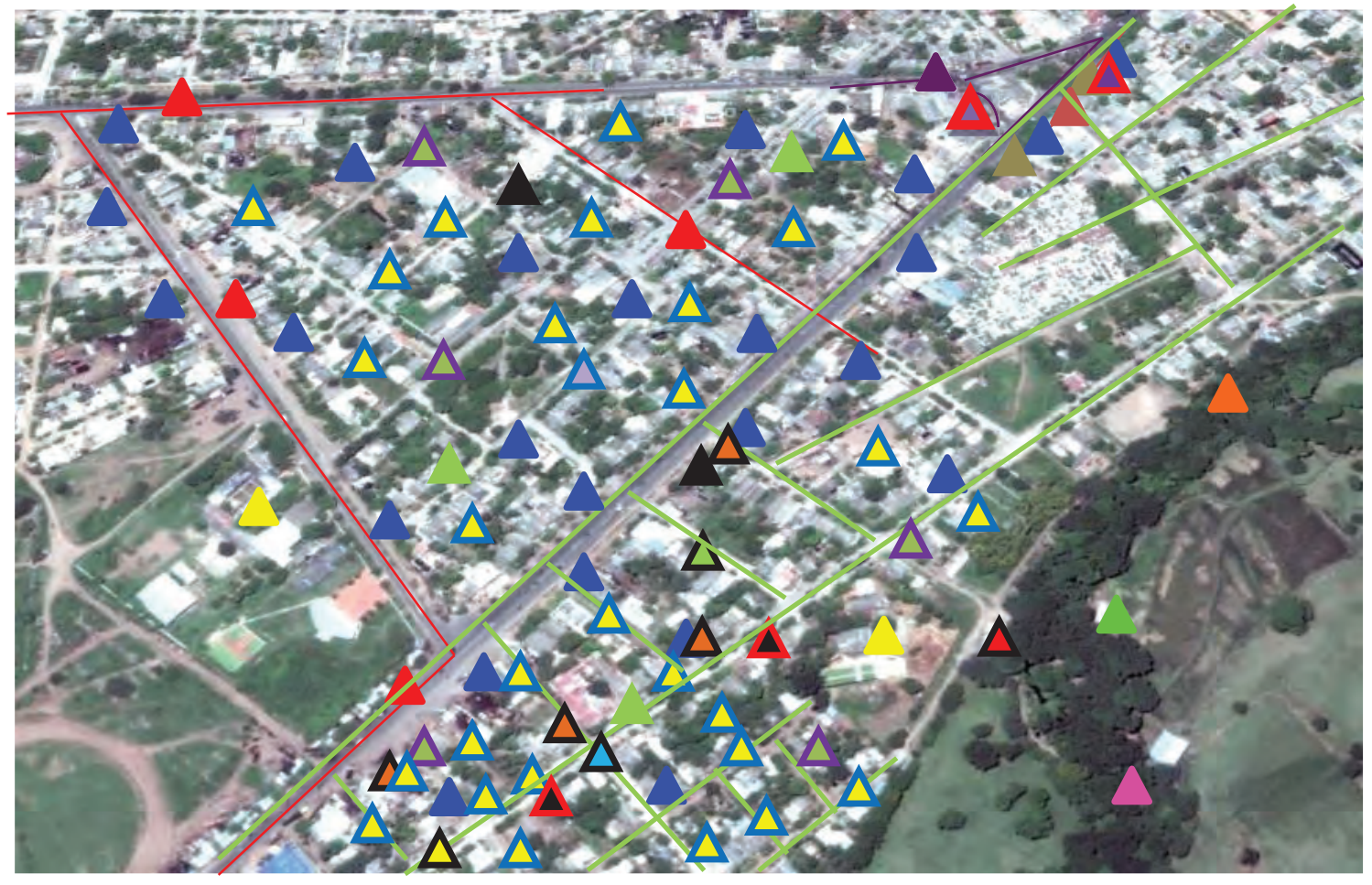

Figura 1. Mapeo aritmético de un sector del municipio de la Paz, Cesar, Colombia. Fotografía tomada con google earth y distribución de triángulos, mediante jornadas de observaciones no participantes.

la zona escogida, en algunos casos, muy diferente a la empleada en el contexto escolar. En la figura 1, se ubicaron las líneas de influencia de una aritmética, por ejemplo, se pueden ver las líneas rojas que son las líneas de influencia de la aritmética del oficio, representado por el triángulo rojo. Estas líneas de influencia es solo una noción que se quiso dar a conocer, pues su real alcance es muy complejo. Como algunos oficios o actividades están anclados en lugares geográficos y las aritméticas al estar sujetas a oficios o actividades (Carraher et al. 1993), éstas, entonces, se anclan también en algunos sectores; por ejemplo, no sería normal vender gasolina de contrabando al lado de un colegio de preescolar o curtir, artesanalmente, cuero distante del río. Para el desarrollo de una actividad u oficio, se necesitan unas condiciones, incluso naturales, como estar al lado del río; geográfica, que las calles sean de fácil acceso para movilizar el carrito de fritos o sociales, por el flujo del intercambio comercial, para que una aritmética responda a esos problemas descritos al principio, de observar, de comparar, de clasificar y de cuantificar. En estos oficios, se comienzan a vincular, asumiendo ciertas tareas, los niños y niñas, a partir de cierta edad, en particular, cuando sus rangos de edad oscilan entre 10 a 12 años, que coincide con los niveles escolares de quinto de primaria hasta séptimo de secundaria, siendo este último, el de la enseñanza de la aritmética, para luego pasar al álgebra, en octavo grado.

La figura 2 presenta una muestra de 21 oficios, que se ubicaron en la figura 1 , que se consideró tiene asociada su propia aritmética.

No se va a profundizar en el papel que juegan las herramientas en cada uno de los oficios, para la construcción o desarrollo de los métodos o algoritmos, para hacer los respectivos cálculos; en Flores (2011) este tema se analiza con las implicaciones descritas. Lo que interesa en este momento, es pasar a otro nivel y es el de establecer los algoritmos u operaciones que se pueden llegar a involucrar en cada una de las aritméticas, descritas en la figura 2; ésta, pretende resaltar los diversos procesos y algoritmos aritméticos que pueden existir en una región en concreto. Además de los escolares, existen procesos aritméticos, como el manejo de billetes y de monedas, el redondeo, el manejo del tiempo, estimaciones de longitudes, combinaciones de patrones, proporcionalidad y comparaciones entre objetos, percepciones y abstracciones; aparecen algoritmos, como el del complemento, la suma larga, tanto de $\mathrm{X}$ por tanto de Y. También advierte sobre otras unidades de medida no convencionales, 


\begin{tabular}{|c|c|c|c|c|c|c|c|c|c|c|c|c|c|}
\hline 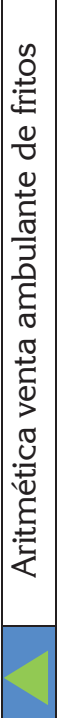 & 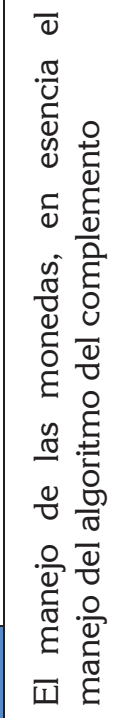 & 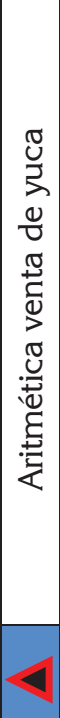 & 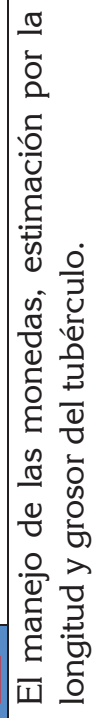 & 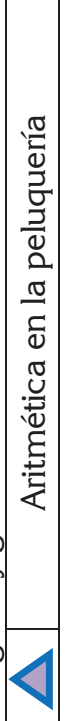 & 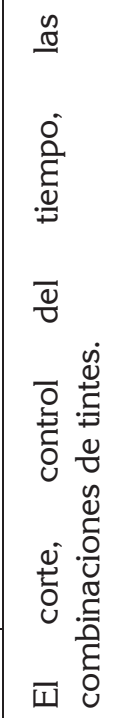 & 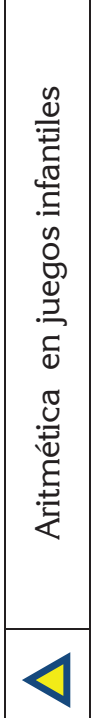 & 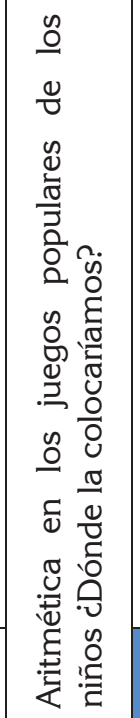 & 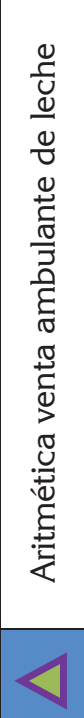 & 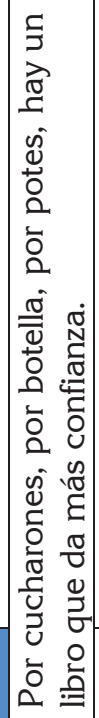 & 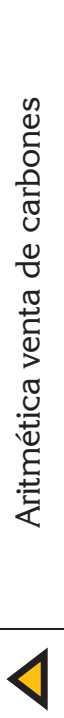 & 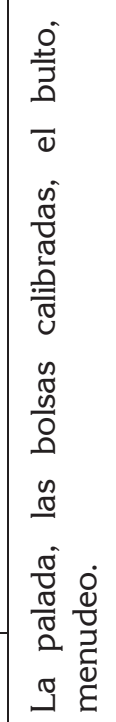 & 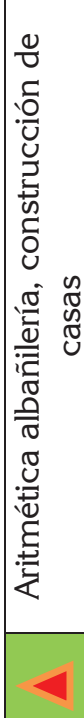 & 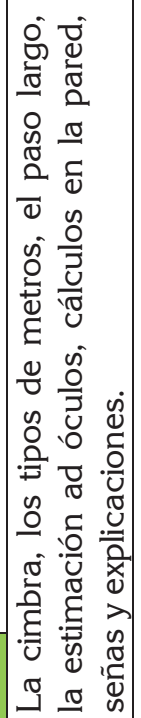 \\
\hline 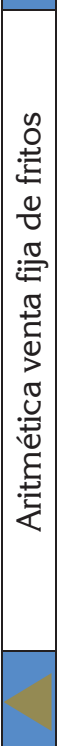 & 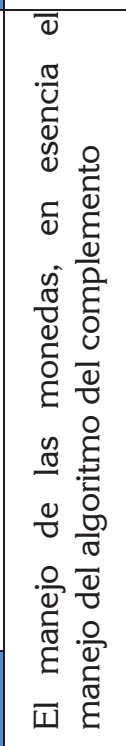 & 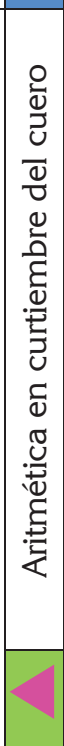 & 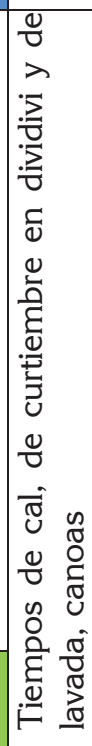 & 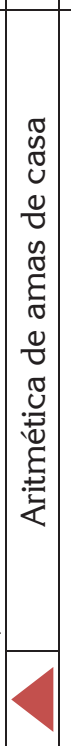 & 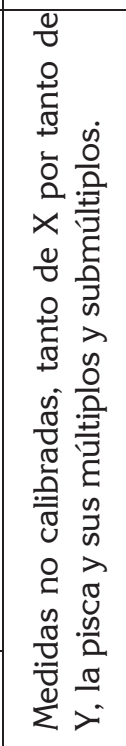 & 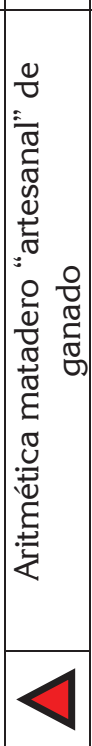 & 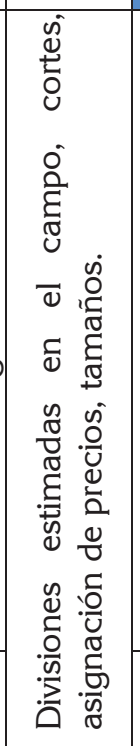 & 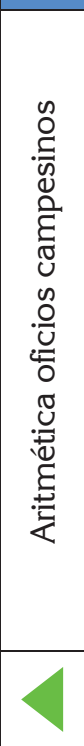 & 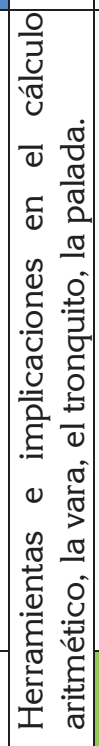 & 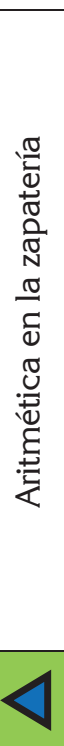 & 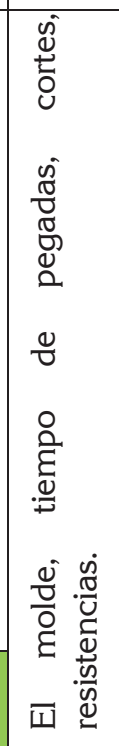 & 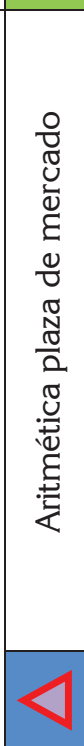 & 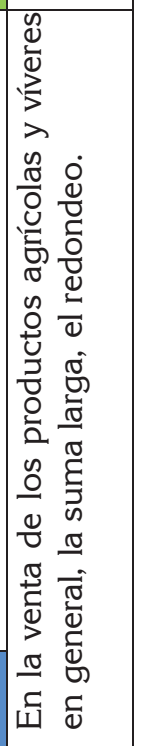 \\
\hline 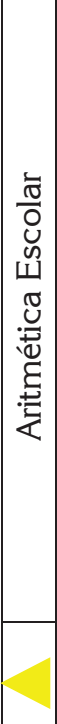 & 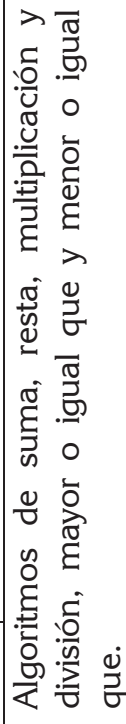 & 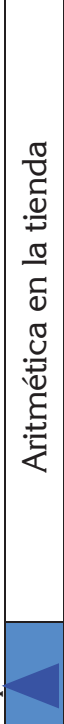 & 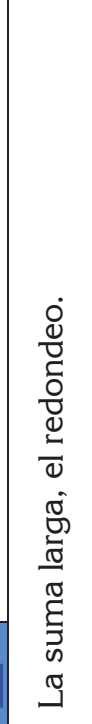 & 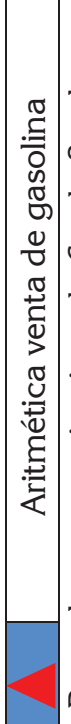 & 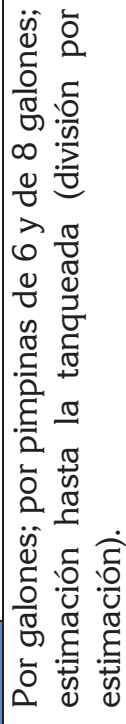 & 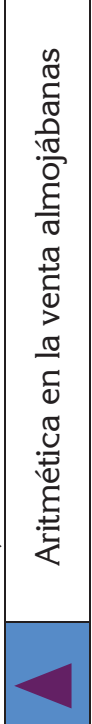 & 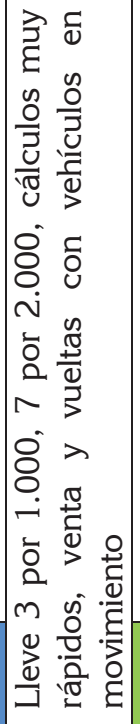 & 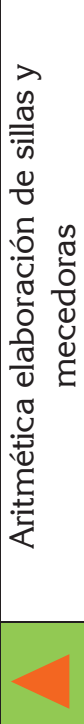 & 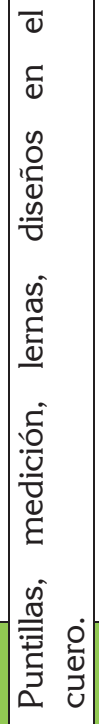 & 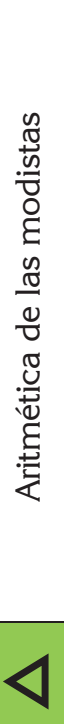 & 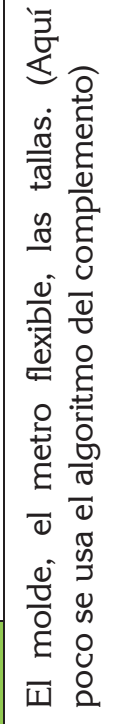 & 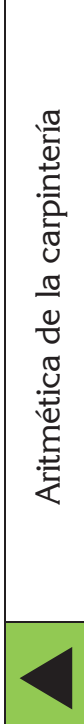 & 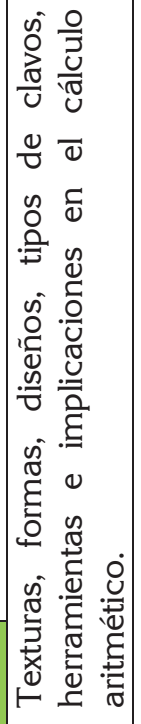 \\
\hline
\end{tabular}


como la palada, el galón o la pimpina, el bulto, la vara, el tronquito, el cucharón, la botella o instrumentos para medir, como la cimbra, el metro flexible o medidas antropométricas, como el paso largo, la cuarta, el geme y la pisca. En la figura 2, se puede identificar, de manera muy sucinta, cómo las comunidades de prácticas crean sus propios procesos y algoritmos aritméticos, para sobrevivir y trascender en el tiempo.

Hay aritméticas que poseen más recursos de cálculo para resolver un problema en concreto, lo cual, complejiza las formas de calcular, algoritmos u operaciones. Cada uno de los oficios, se caracteriza, entonces, por tener un ambiente, un contexto particular asociado a la aritmética. En estos contextos las personas asumen roles distintos, manualidades, técnicas, artes, fuerza física o solo esfuerzo mental. Esto es lo que implica el contexto y la actividad en sí, donde para algunos niños es imposible asumir la aritmética, sea por falta de fuerza, de desarrollo psicomotriz o de habilidades cognitiva, que aún no ha desarrollado.

Se analizaron algunos aspectos que se pudieron notar en los oficios, en particular, en sus algoritmos. En todos los oficios, cuya celda del triángulo aparece rellena en azul, se emplea el algoritmo del complemento, para dar el cambio o el vuelto, que no es el de la diferencia escolar, siendo esta otra operación aritmética diferente a las propuestas en el contexto escolar. Mariño (1985; 1990) hizo investigaciones al respecto, en particular, sobre formas de calcular, aritméticamente los adultos, en sectores populares. Este algoritmo del complemento no es de manejo de niños sino de adultos y jóvenes, que están familiarizados con el manejo del dinero, en particular, de todas las denominaciones de los billetes.

Un ejemplo del algoritmo del complemento: alguien paga una deuda de $\$ 17.500$ con un billete de $\$ 50.000$, el vendedor da los vueltos así: 500 para 18, 2 para 20,10 para 40 y 10 para 50 . Basta con preguntarle a una persona que emplea este algoritmo cuánto tenía que dar y se notará que para ellos no existe lo que llamamos diferencia. $10-8=2$, en este caso, 2 es la diferencia en la escuela, en el contexto escolar, pero en aquellos oficios que emplean este procedimiento del complemento, sobre el 2 , no hay conciencia, como tampoco sobre la diferencia, es decir, el 2 no existe en el cálculo mental cuando se emplea este tipo de algoritmo. Al vendedor se le puede preguntar ċcuánto tenías que darme? Y en nuestros trabajo de campo encontramos dos reacciones: 1. Regularmente preguntaban ¿cuánto te di?, ¿cuánto tenía que darte?, ¿cuánto tienes ahí? o 2; procedían a quitar el vuelto o el cambio. En algunos casos, recurrían a la calculadora, si existía; otros, iban al cálculo mental, pero empleando el algoritmo escolar; otros, escribían imaginariamente sobre una superficie y se notaba el empleo del algoritmo escolar; en fin, todas estas segundas estrategias de cálculos implicaba más tiempo y, en algunos caso, más dificultad para la persona o vendedor.

En la tabla 1, en otra investigación en la ciudad de Barranquilla, con dos de mis estudiantes, Romario Palacios y Freddy Ramírez, que se hizo sobre el empleo de algoritmos y operaciones aritméticas de vendedores informales en esta ciudad, cuyas actividades $\mathrm{u}$ oficios son similares a las encontradas en la región descrita, se estableció una sistematización de los cálculos de varios vendedores informales, en la plaza de mercado. En este caso, el interés radica en mostrar la relación entre los cálculos mentales empleados por los niños, jóvenes y adultos escolarizados y adultos no escolarizados, sin importar la actividad en que se desempeñe; es por ello, que los principales referentes teóricos que se tienen en dicha investigación son Gómez (1994), Delprato (2005), De Marinis (2008), Crespo et al. (2009), Gómez (2010) y Guerrero (2011). Entre estos referentes, los trabajos de Bernardo Gómez son los referentes teóricos principales en dicha investigación, dado que ha analizado a profundidad el cálculo mental.

Se encontró que en el caso de la suma son más recursivos en cuanto a proceso y algoritmos. En el caso de la resta, los algoritmos mentales de los vendedores consisten, básicamente, en sustituir algunas o todas las partes de la resta por una cifra más cómoda en el sustraendo o minuendo, por medio de un redondeo de cifras, ya sea en decenas o centenas. Para la multiplicación, la propiedad distributiva fue la más utilizada y la de mayor frecuencia en las respuestas. La hipótesis aquí es que la propiedad distributiva tiene su origen en los contextos no escolarizados; Gómez (1995) ya lo había notado, en particular, en lo que él llamó vertimientos de los contextos a los textos de matemáticas. En el caso de la división, se obtuvieron pocas respuestas en comparación con las otras operaciones, donde sí hubo varios resultados y se debe a que como esta operación, al igual que la resta, no es tan utilizada por ellos en sus oficios. Se puede observar, que en estos procesos y algoritmos, al ser de carácter mental, existe mucha creatividad. En la tabla 1, se sistematizaron los cálculos de tres vendedores.

Los datos en la tabla 1, que representan algoritmos aritméticos de vendedores ambulantes o de la plaza de mercado de Barranquilla, sirvieron para hacer comparaciones con los resultados obtenidos en el mapeo aritmético proveniente del municipio de la Paz y estos coincidieron. Se considera que esta coincidencia, se debe a que las poblaciones escogidas se desempañan en oficios similares o iguales, es decir, se encontró que en dos regiones diferentes de la costa caribe colombiana los tipos de cálculo mental son similares y que están sujetos a la experiencia de cada individuo con el oficio; sin embargo, se pudo establecer que al insistirle a una persona que se negaba a calcular mentalmente, lo cual, cada 


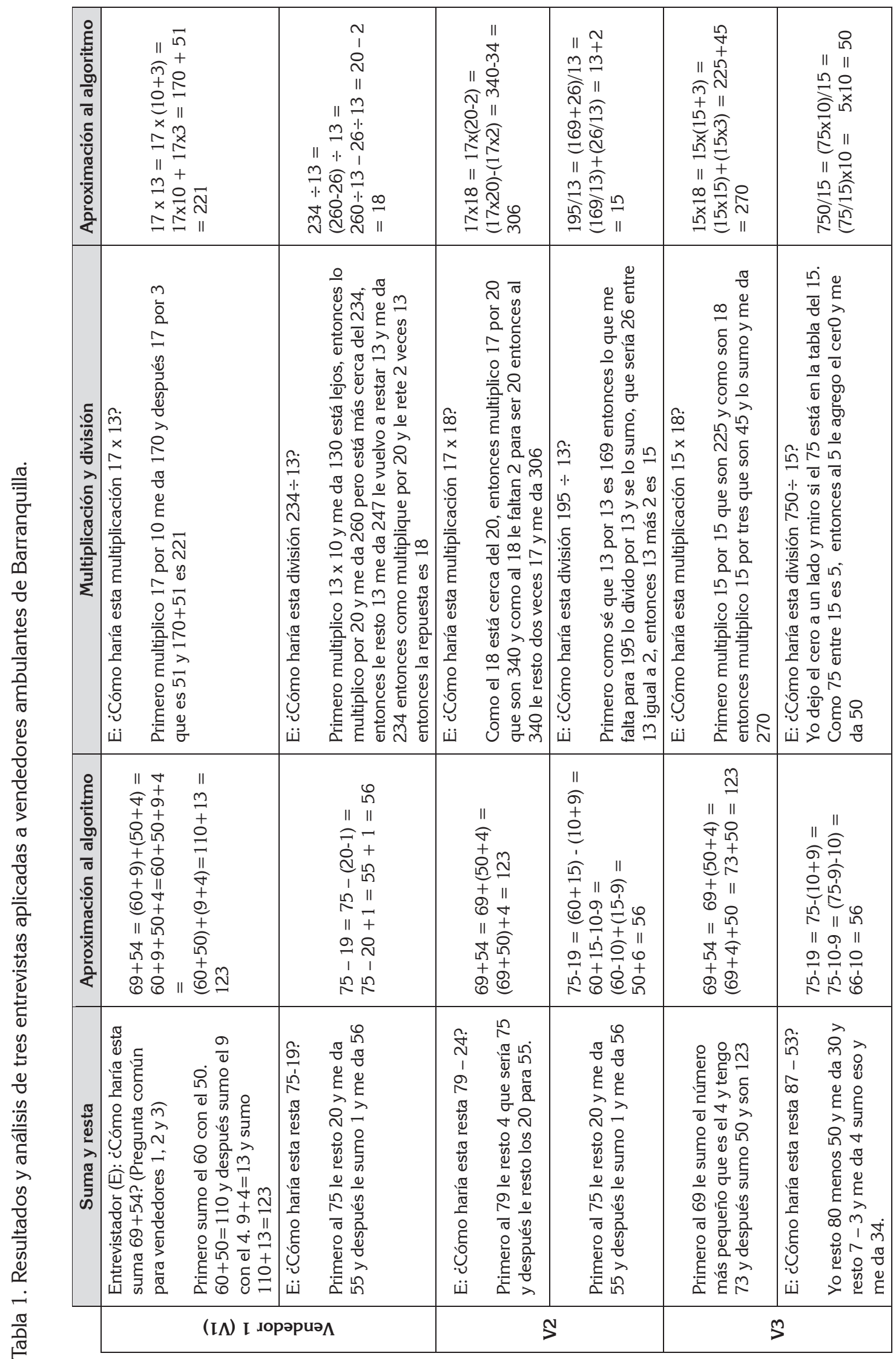


día es más difícil de lograr, como objetivo pedagógico, pues en su lugar, se emplean objetos manipulativos, como calculadora o el celular, tiende a aplicar las mismas descomposiciones o procedimientos. Un $\mathrm{PhD}$. en matemáticas tiende a calcular, mentalmente, de manera similar que una persona que vende fritos en las calles; de hecho, este último lo hace más rápido que el primero. La importancia del cálculo mental es que es la base de la comprensión de los procesos y de los algoritmos aritméticos escritos.

En la figura 2, las celdas de los triángulos que están rellenas en verde, las herramientas que se han desarrollado en cada uno de estos oficios juegan un papel esencial en los algoritmos u operaciones empleados.

Estas herramientas son esenciales para el desarrollo del conocimiento y el mismo aprendizaje, pero aquí se encontró una particularidad, que no hay una intercepción entre los procesos o algoritmos de los oficios, cuyas celdas están rellenas con azul, con respecto a las celdas rellenas en verde. El algoritmo del complemento no se intercepta con los algoritmos u operaciones de oficios, donde se emplean varias herramientas, es decir, el algoritmo del complemento que, esencialmente, se emplea en los oficios de menudeo o intercambio comercial rápido, no se pudo notar que se empleara en los oficios, cuyo uso de herramientas es clave. Se consideró que, lo anterior, se debe a que en las actividades del menudeo el comercio o intercambio del producto es más rápido y, por ende, no se necesitan tantas herramientas, sino cálculos mentales ágiles.

De la presente investigación, se puede concluir que en los contextos que están por fuera del ámbito escolar, representados por los oficios, no se generan tensiones en la compresión entre aritméticas, algoritmos u operaciones, de los diversos grupos culturales, excepto cuando esta comunicación se pretende dar con el escolar, es decir, no se produce la intercomprensión de lógicas. Las aritméticas provenientes de oficios no escolares, no quieren entre sí imponer sus lógicas y acciones de cálculo, procesos o algoritmos y operaciones, lo importante es que se puedan comunicar. No tienen un agente o un profesor que supervise esta imposición, que se camufla en la palabra competencia. Esta condición de respeto, le permite al pueblo poderse comunicar aritméticamente, así, las aritméticas tienen sentido y significado para las personas (Chahine \& Naresh, 2013) y fragilidad, ya que pueden desaparecer si deja de existir el oficio, pues la aritmética no siempre se desarrolla por el individuo sino por las pequeñas comunidades que se aglutina por medio del oficio (Aroca, 2013). Si aún se duda de las tensiones que se generan por medio de esta comprensión interlógica de aritméticas, se podrá notar que los encuentros matemáticos no han sido en la historia de la humanidad pacifista. D'Ambrosio (2012) plantea este tema de la paz y su relación con la forma de pensamiento más difundida en el mundo, el matemático. Sería extraño encontrar un profesor de matemáticas maltratando a un niño o niña, porque no se aprendió el algoritmo u operación aritméticos, pero sí es posible escucharle decir así no es, ese así no es y puede significar que los cálculos u operaciones que emplea el padre o la madre, o todo el núcleo familiar, que ellos emplean casi a diario para sobrevivir, no sirven. Aunque esto también es recíproco, el tendero dirá algo propio si no le da el mismo resultado, empleando el algoritmo escolar. Se precisa que -el no sirven-, solo sería válido si no da el resultado correcto para los que no están involucrados; una apuesta compleja en una situación didáctica.

Si se mira ahora lo que sucede en la mayoría de las escuelas, se notará que se sigue enseñando a calcular aritméticamente, a través de un único algoritmo para cada operación, es decir, no hay un cálculo flexible (Gómez, 2010). Al enfocarse, de esta manera, se produce una tensión, pues las personas, los estudiantes, cuentan con otras formas de calcular y llegan a tener una comprensión profunda de los números, de las cantidades, de las magnitudes, el papel que juegan en los oficios, su diversidad algorítmica y procedimental. Lo curioso es que el mismo profesor de matemáticas no está excepto de esto; si hay una conciencia en ambas direcciones, entre agentes de los oficios y la escuela, desde la escuela que no vena las aritméticas de los oficios, como ejemplos de lo escolar y los agentes de los oficios no vean lo escolar, como impositiva, tal vez esta conciencia puede permitir una intercomprensión de lógicas de las aritméticas escolares o provenientes de oficios. Si lo primero se logra y se comprende, las vinculaciones de los niños y niñas a estos oficios, entonces, se podrá construir un significado sobre el real alcance de la frase de que la matemática tiene sentido si se vincula con la realidad.

Por último, la educación matemática, para aproximarse al respeto que caracteriza la intercomprensión de lógicas de las aritméticas de oficios, necesita de la enculturación matemática del currículo (Bishop, 1999), teniendo como actores fundamentales a los profesores de matemáticas, sensibles a sus entornos próximos, donde éste labora, los cuales, son fecundos en aritméticas. Es solo una cuestión de ir con otros ojos a la calle.

Agradecimientos: A los profesores Carlos Eduardo Vasco y Bruno D'Amore, por revisar este texto en sus primeras versiones, por sus observaciones y recomendación de bibliografía. Conflictos de intereses: El manuscrito fue preparado y revisado por el autor, quien declara ser el único autor y que no existe conflicto de intereses, que ponga en riesgo la validez de los resultados presentados. 


\section{BIBLIOGRAFÍA}

1. AROCA, A. 2013. Cálculos aritméticos en una práctica cultural. EAE, España. 64p.

2. BISHOP, A. 1999. Enculturación matemática: La educación matemática desde una perspectiva cultural. Ed. Ibérica S.A. Paídos, SAICF, Buenos Aires. 239p.

3. BOYER, C. 1974. História da Matemática. Editora da Universidade de São Paulo, Brazil. 252p.

4. CARRAHER, T.; CARRAHER, D.; SCHLIELMANN, A. 1993. Na vida dez, na escola zero. $3^{\text {a }}$. ed. Cortez, Brasil. 184p.

5. CHAHINE, I.C.; NARESH, N. 2013. Mapping the cognitive competencies of street vendors and bus conductors: A cross-cultural study of workplace mathematics. Rev. Latinoam. Etnomatem. (Colombia). 6(3):7-35.

6. CRESPO, C.; FARFÁN, R.; LEZAMA, J. 2009. Algunas características de las argumentaciones y la matemática en escenarios sin influencia aristotélica. Rev. Latinoam. Invest. Matemát. Educat. (México). 12(1):2966.

7. D'AMBROSIO, U. 2000. Las dimensiones políticas y educacionales de la etnomatemática. En:Martinón, A.; (ed.). Las matemáticas del siglo XX, una mirada en 101 artículos. NIVOLA Libros y Ediciones, España, p.439-444.

8. D'AMBROSIO, U. 2011. Etnomatemática. Elo entre as tradições e a modernidade. Autentica Editora, Belo Horizonte, 110p.

9. D'AMBROSIO, U. 2012. The program ethnomathematics: theoretical basis and the dynamics of cultural encounters. Cosmopolis. J. Cosmopolitics. 3(4):1341.

10. De MARINIS, S. 2008. Matemática. Cálculo mental con números naturales para el docente. Ministerio de Educación Gobierno de la ciudad de Buenos Aires, Buenos Aires, 56p.

11. DELPRATO, M. 2005. Educación de adultos. ¿Saberes matemáticos previos o saberes previos a los matemáticos? Acta Latinoam. Matemát. Educat. (México). 18:51-56.
12. DESLAURIERS, J. 2005. Investigación cualitativa. Guía práctica. Ed. Papiro, Colombia, 142p.

13. FLORES, F. 2011. Enciclopedia de las tecnologías Rotas. El humanista como Ingeniero. Libro Primero. Media - Tryck, Suecia, 134p.

14. GOETZ, J.; LeCOMPTE, M. 1998. Etnografía y Diseño Cualitativo en investigación educativa. Ed. Morata, Madrid), 280p.

15. GÓMEZ, B. 1994. Tipología de los errores en el cálculo mental. Un estudio en el contexto educativo. Enseñanza Ciencias. 13(3):313-325.

16. GÓMEZ, B. 1995. Los métodos de cálculo mental vertidos por tradición reflejados en los libros de aritmética. Uno: Rev. Didáctica Matemát. (España). 2(5):91101.

17. GÓMEZ, B. 2010. El cálculo flexible. En: Las matemáticas su enseñanza y aprendizaje. Org. Velasco, M.C.; Mejía, M.F. p.17-31. Unidad de Artes Gráficas - Facultad de Humanidades, Cali, 150p.

18. GUERRERO, F. 2011. La importancia de las estrategias del cálculo mental en las operaciones matemáticas básica. Rev. Digital Innov. Experien. Educat.40:1-7. Disponible desde Internet en: http://www.csi-csif.es/ andalucia/modules/mod_ense/revista/pdf/Numero_40/FRANCISCO\%20JĀVIER_GUERRERO_1.pdf (con acceso el 02/10,2014).

19. HODGKIN, L. 2005. A history of mathematics. From Mesopotamia to modernity. Oxford University Press, New York, 283p.

20. MARIÑO, G. 1985. Cómo opera matemáticamente el adulto del sector popular, constataciones y propuestas. Dimensión Educativa, Bogotá, 161p.

21. MARIÑO, G. 1990. La resta desde los sectores populares. En: Centro Laubach de educación popular básica de adultos. p. 151-157. Consejo de educación de adultos de América Latina. La enseñanza de la matemática con los adultos de los sectores populares: Experiencias e investigaciones. Dimensión Educativa, Bogotá, 184p.

22. MORSE, H.; BOTTORFF, J.; ZIMMERMAN, E. 2007. Asuntos críticos en los métodos de investigación cualitativa. Ed. Universidad de Antioquia, Colombia, $185 p$. 
23. SAXE, G.B. 1988. The mathematics of child street vendors. Child Developm. (Estados Unidos). 59:14151425.

24. SAXE, G.B. 1991. Venditori ambulanti e conoscenze matematiche. Età Evolutiva. (Italia). 40:3-16.

25. SEAQUIST, C.; SESHAIYER, P.; CROWLEY, D. 2005. Calculation across cultures and history. Texas College Mathemat. J. (Estados Unidos). 1(1):15-31.

26. STRAUSS, A.; CORBIN, J. 2002. Bases de la investigación cualitativa. Técnicas y procedimientos para desarrollar la teoría fundamentada. Primera edición en español, Medellín: Editorial Universidad de Antioquia. 340p.

27. TAYLOR, T. 1816. Theoretic arithmetic. Printed for the author, London, 252p.

28. WITTGENSTEIN, L. 1997. Observaciones sobre los fundamentos de la matemática, Madrid: Alianza, 384p.

Recibido: Julio 31 de 2015

Aceptado: Octubre 6 de 2015

Cómo citar:

Aroca, A. 2015. Aritméticas en un municipio del nororiente colombiano. Rev. U.D.C.A Act. \& Div. Cient. 18(2): 553-561. 